\title{
Disposition of Cannabidiol Metabolites in Serum and Urine from Healthy Individuals Treated with Pharmaceutical Preparations of Medical Cannabis
}

\author{
Ana Pilar Pérez-Acevedo ${ }^{1,2, \dagger}$, Francesco Paolo Busardò ${ }^{3, *}$, Roberta Pacifici ${ }^{4}$, Giulio Mannocchi ${ }^{5}$,

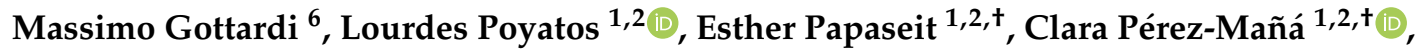 \\ Soraya Martin ${ }^{1,2}$, Annagiulia Di Trana ${ }^{3}$, Simona Pichini ${ }^{4, \ddagger}$ and Magí Farré ${ }^{1,2, \ddagger(1)}$ \\ 1 Clinical Pharmacology Unit, Hospital Universitari Germans Trias i Pujol and Institut de Recerca Germans \\ Trias i Pujol (HUGTiP-IGTP), 08916 Badalona, Spain; appereza.germanstrias@gencat.cat (A.P.P.-A.); \\ lpoyatos@igtp.cat (L.P.); epapaseit.germanstrias@gencat.cat (E.P.); cperezm.mn.ics@gencat.cat (C.P.-M.); \\ smartins.mn.ics@gencat.cat (S.M.); mfarre.germanstrias@gencat.cat (M.F.) \\ 2 Department of Pharmacology, Therapeutics and Toxicology, Universitat Autònoma de Barcelona, \\ Cerdanyola del Vallés, 08193 Bellaterra, Spain \\ 3 Department of Excellence-Biomedical Sciences and Public Health, Università Politecnica delle Marche, \\ 60121 Ancona, Italy; annagiulia.ditrana@gmail.com \\ 4 National Centre on Addiction and Doping, Istituto Superiore di Sanità, 00161 Rome, Italy; \\ roberta.pacifici@iss.it (R.P.); simona.pichini@iss.it (S.P.) \\ 5 School of Law, University of Camerino, 62032 Camerino, Italy; giulio.mannocchi@unicam.it \\ 6 Comedical srl, 38123 Trento, Italy; massimo.gottardi@comedical.biz \\ * Correspondence: fra.busardo@libero.it; Tel.: +39-0715-9650-43 \\ + These authors share first authorship. \\ $\ddagger$ These two authors equally contributed as Senior Investigators.
}

Received: 29 November 2020; Accepted: 11 December 2020; Published: 12 December 2020

\begin{abstract}
The use of cannabis flowering tops with standardized amounts of active phytocannabinoids was recently authorized in several countries to treat several painful pathological conditions. The acute pharmacological effects and disposition of $\Delta$-9-tetrahydrocannabinol (THC), cannabidiol (CBD), their acidic precursors and THC metabolites after oil and decoction administration have been already described. In this study, the disposition of CBD metabolites: 7-carboxy-cannabidiol (7-COOH-CBD), 7-hydroxycannabidiol (7-OH-CBD), 6- $\alpha$-hydroxycannabidiol (6- $\alpha$-OH-CBD), and $6-\beta$-hydroxycannabidiol $(6-\beta-\mathrm{OH}-\mathrm{CBD})$ in the serum and urine of healthy volunteers was presented. Thirteen healthy volunteers were administered $100 \mathrm{~mL}$ of cannabis decoction in the first experimental session and, after 15 days of washout, $0.45 \mathrm{~mL}$ of oil. Serum and urine samples were collected at different time points, and the CBD metabolites were quantified by ultra-high-performance liquid chromatography-tandem mass spectrometry. The most abundant serum metabolite was 7-COOH-CBD, followed by 7-OH-CBD, $6-\beta-\mathrm{OH}-\mathrm{CBD}$, and6- $\alpha-\mathrm{OH}-\mathrm{CBD}$, after decoction and oil. Both 7-OH-CBD and the $6-\alpha-\mathrm{OH}-\mathrm{CBD}$ showed similar pharmacokinetic properties following administration of both cannabis preparations, whereas $7-\mathrm{COOH}$ and $6-\alpha-\mathrm{OH}-\mathrm{CBD}$ displayed a significant higher bioavailability after decoction consumption. All CBD metabolites were similarly excreted after oil and decoction intake apart from $6-\alpha-\mathrm{OH}-\mathrm{CBD}$, which had a significantly lower excretion after oil administration. The pharmacokinetic characterization of CBD metabolites is crucial for clinical practice since the cannabis herbal preparations are increasingly used for several pathological conditions.
\end{abstract}

Keywords: cannabis; cannabidiol; cannabidiol metabolism; medical cannabis 


\section{Introduction}

$\Delta$-9-tetrahydrocannabinol (THC) and cannabidiol (CBD) are the most abundant and pharmacologically active phytocannabinoids of the Cannabis sativa L. plant [1-5]. Whereas THC produces psychotropic and addictive effects, being a partial agonist at $\mathrm{CB} 1$ and $\mathrm{CB} 2$ receptors, $\mathrm{CBD}$, as an antagonist, presents anxiolytic, myorelaxant, and antiepileptic properties without psychotropic or addictive effects [6-8].

Unlike recreational cannabis, which contains elevated proportions of THC, medical cannabis contains both THC and CBD at different proportions, together with terpenoids and other minor phytocannabinoids. It has been demonstrated that all these constituents are necessary to provide the best pharmacological effects for the treatment of chronic neurogenic pain, multiple sclerosis with muscle spasticity, amyotrophic lateral sclerosis, cachexia, cancer, glaucoma, Acquired immunodeficiency syndrome, Crohn disease, post-traumatic stress disorder, resistant epilepsy, and seizures $[6,8]$.

Since cannabis decoction and oil are the most used medical cannabis herbal preparations, we recently investigated the disposition of THC, CBD, their acidic precursors $(\Delta$-9-tetrahydrocannabinolic acid A-THCA-A and cannabidiolic acid-CBDA) and THC principal metabolites in the serum, oral fluid, sweat patch, and urine of healthy individuals treated with these two products [9].

Indeed, it was the first time that the pharmacokinetics of phytocannabinoids present in medical cannabis was explored in healthy subjects, since two previous studies involved children and young adults with drug-resistant epilepsy [10,11] and medication-overuse headache patients [12].

At that time, only THC metabolites could be detected and their kinetics investigated in our biological samples, while CBD metabolites could not be measured due to the unavailability of chemical standards. However, we believed that the examination of CBD metabolites should have been essential to better understand the mechanisms underlying the effects of different preparations of medical cannabis and of CDB-based pharmaceutical products.

Once CBD metabolites became commercially available, we investigated the disposition and pharmacokinetics of cannabidiol-7-oic acid (7-COOH-CBD), 7-hydroxycannabidiol (7-OH-CBD), 6-alpha-hydroxycannabidiol (6- $\alpha-\mathrm{OH}-\mathrm{CBD})$, and 6-beta-hydroxycannabidiol (6- $\beta-\mathrm{OH}-\mathrm{CBD})$, in serum and urine samples stored from the previous study concerning healthy individuals treated with a single administration of cannabis decoction and cannabis oil containing similar amounts of phytocannabinoids.

\section{Results}

\subsection{Subjects and Study Design}

Fourteen subjects were initially recruited to complete the study. However, one male showed high serum concentration of THC metabolites at baseline time $(0 \mathrm{~h})$ and was therefore excluded from the study, so that finally, 13 subjects, 11 males and 2 females, completed the two experimental sessions. As for men, the mean age was $24.2 \pm 3.3$ years (range 19-32 years); mean weight was $76.3 \pm 11.9 \mathrm{~kg}$ (range 65-105 kg), and mean height was $1.8 \pm 0.2 \mathrm{~m}$ (range 1.7-1.9 m). The women were 23 and 25 years old, weighed 56 and $63 \mathrm{~kg}$, and were 1.59 and $1.67 \mathrm{~m}$ tall, respectively. Globally, the subjects had an average onset of cannabis use at $17.5 \pm 1.7$ years (range 14-19 years), with a mean of 30 cannabis sessions in the last year and a mean of 0.90 joints by consumption. All subjects had a history of oral cannabis use at least once in their life, $53 \%$ of subjects had ingested hallucinogenic mushrooms on some occasion, and 38\% had ever used cocaine, ecstasy, amphetamines, or MDMA. Regarding smoking, $46 \%$ of subjects were tobacco smokers and all had experience with alcohol intake, with a mean consumption of 1.19 alcoholic units/day. The thirteen subjects were administered $100 \mathrm{~mL}$ of the FM2 cannabis decoction containing $0.3 \pm 0.12 \mathrm{mg}$ THC, $1.2 \pm 0.4 \mathrm{mg}$ THCA-A, $0.7 \pm 0.4 \mathrm{mg}$ of CBD, and $4.4 \pm 0.6 \mathrm{mg}$ of CBDA. After wash out period, they were administered $0.45 \mathrm{~mL}$ of FM2 cannabis oil containing $1.0 \pm 0.2 \mathrm{mg}$ THC, $1.4 \pm 0.3 \mathrm{mg}$ THCA-A, $0.9 \pm 0.2 \mathrm{mg}$ of CBD and $2.8 \pm 0.4 \mathrm{mg}$ of CBDA. Significantly higher amounts of THC and CBD were observed in cannabis oil and significantly higher amounts of CBDA in cannabis decoction after preparation. We studied the disposition of CBD metabolites in subjects' serum and urine samples but did not investigate their presence in oral fluid 
or sweat since we previously demonstrated that THC metabolites were not excreted in these latter biological matrices [12], and preliminary analyses for CBD metabolites in a couple of samples also confirmed the absence of these metabolites.

\subsection{Concentration-Time Profiles and Pharmacokinetics of CBD Metabolites in Serum after Decoction and Oil Administration}

Figure 1 shows the mean time course of $6-\alpha-\mathrm{OH}-\mathrm{CBD}, 6-\beta-\mathrm{OH}-\mathrm{CBD}, 7-\mathrm{OH}-\mathrm{CBD}$, and $7-\mathrm{COOH}-\mathrm{CBD}$ concentrations in serum following the administration of decoction and oil.

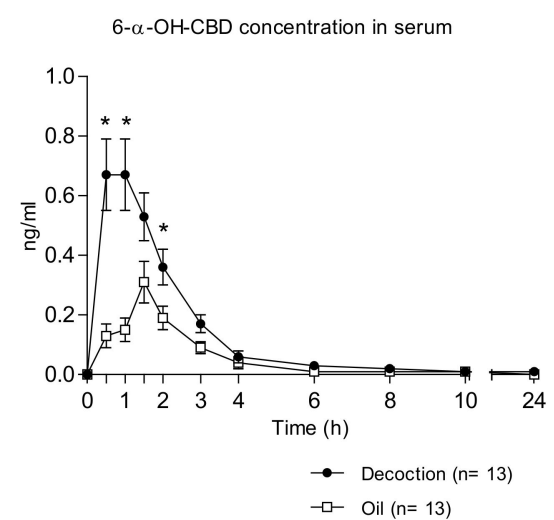

7-OH-CBD concentration in serum

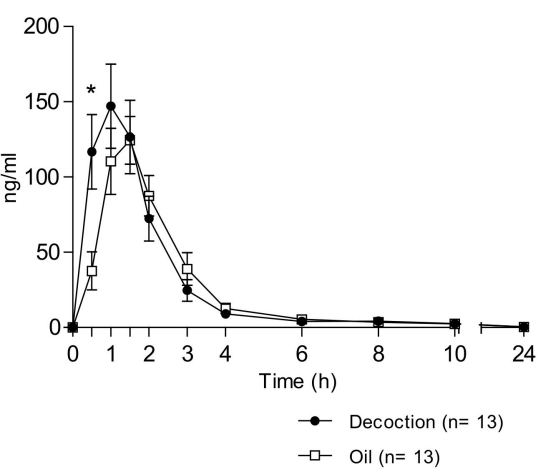

$6-\beta-\mathrm{OH}-\mathrm{CBD}$ concentration in serum

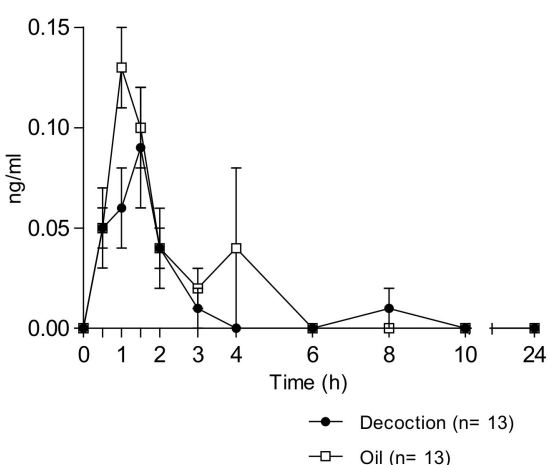

7-COOH-CBD concentration in serum

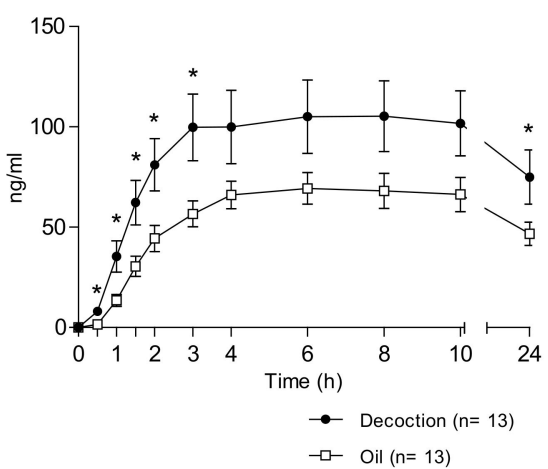

Figure 1. Mean time course of 6-alpha-hydroxycannabidiol (6- $\alpha$-OH-CBD), 6-beta-hydroxycannabidiol (6- $\beta$-OH-CBD), 7-hydroxycannabidiol (7-OH-CBD), and cannabidiol-7-oic acid (7-COOH-CBD) concentrations in serum following the administration of cannabis decoction and oil $(n=13$, mean values \pm standard error). The time course of analytes' concentrations was analyzed employing a two-way, repeated-measures ANOVA with formulation and time as factors. When treatment condition or the treatment condition $\times$ time interaction was statistically significant, a Student's t-test for paired samples was performed. The symbol * shows significant differences between decoction and oil concentration.

It can be noted that $6-\beta-\mathrm{OH}-\mathrm{CBD}$ showed the lowest serum concentrations among the detected metabolites, with a peak concentration $1.5 \mathrm{~h}$ after decoction intake comparable to the one measured $1 \mathrm{~h}$ after oil intake $(0.12 \pm 0.08$ and $0.17 \pm 0.11 \mathrm{ng} / \mathrm{mL}$, respectively). Consequently, the area under the concentration-time curve at 10 and $24 \mathrm{~h}\left(\mathrm{AUC}_{0-10 \mathrm{~h}}\right.$ and the $\left.\mathrm{AUC}_{0-24 \mathrm{~h}}\right)$ of this metabolite were comparable following the administration of both formulations (Table 1). Moreover, 7-OH-CBD presented a similar time course $\left(\mathrm{C}_{\max }, \mathrm{AUC}_{0-10 \mathrm{~h}}, \mathrm{AUC}_{0-24 \mathrm{~h}}\right.$ and elimination profiles) in serum after both decoction and oil consumption; but differently from $6-\beta-\mathrm{OH}-\mathrm{CBD}$, it was the CBD metabolite with the highest peak concentration in serum $\left(C_{\max } 159.93 \pm 101.75\right.$ for decoction and $151.45 \pm 58.81$ for oil) (Table 1). 
Table 1. Pharmacokinetic parameters of cannabidiol (CBD) metabolites in serum of healthy volunteers after administration of $100 \mathrm{~mL}$ cannabis decoction and $0.45 \mathrm{~mL}$ cannabis oil $(n=13$; $\mathrm{SD}=$ standard deviation).

\begin{tabular}{|c|c|c|c|}
\hline Parameters & Cannabis Decoction (Mean \pm SD) & Cannabis Oil (Mean \pm SD) & $p$ Value \\
\hline \multicolumn{4}{|c|}{$6-\alpha-\mathrm{OH}-\mathrm{CBD}$} \\
\hline $\mathrm{C}_{\max }(\mathrm{ng} / \mathrm{mL})$ & $0.80 \pm 0.41$ & $0.42 \pm 0.18$ & 0.004 \\
\hline $\mathrm{T}_{\max }(\mathrm{h})$ & $1.0(0.5-2)$ & $1.5(0.5-3)$ & 0.115 \\
\hline $\mathrm{AUC}_{0-10 \mathrm{~h}}(\mathrm{ng} / \mathrm{mL} \cdot \mathrm{h})$ & $1.59 \pm 0.85$ & $0.68 \pm 0.33$ & 0.005 \\
\hline $\mathrm{AUC}_{0-24 \mathrm{~h}}(\mathrm{ng} / \mathrm{mL} \cdot \mathrm{h})$ & $1.69 \pm 0.84$ & $0.78 \pm 0.47$ & 0.006 \\
\hline $\mathrm{K}_{\mathrm{e}}\left(\mathrm{h}^{-1}\right)$ & $0.39 \pm 0.35$ & $0.55 \pm 0.34$ & 0.421 \\
\hline $\mathrm{t}_{1 / 2}(\mathrm{~h})$ & $4.62 \pm 5.37$ & $2.37 \pm 2.65$ & 0.349 \\
\hline \multicolumn{4}{|c|}{$6-\beta-\mathrm{OH}-\mathrm{CBD}{ }^{\S}$} \\
\hline $\mathrm{C}_{\max }(\mathrm{ng} / \mathrm{mL})$ & $0.12 \pm 0.08$ & $0.17 \pm 0.11$ & 0.067 \\
\hline $\mathrm{T}_{\max }($ hour$)$ & $1.5(0.5-3)$ & $1.0(0.5-4)$ & 1.000 \\
\hline $\mathrm{AUC}_{0-10 \mathrm{~h}}(\mathrm{ng} / \mathrm{mL} \cdot \mathrm{h})$ & $0.16 \pm 0.10$ & $0.25 \pm 0.29$ & 0.223 \\
\hline $\mathrm{AUC}_{0-24 \mathrm{~h}}(\mathrm{ng} / \mathrm{mL} \cdot \mathrm{h})$ & $0.17 \pm 0.11$ & $0.26 \pm 0.29$ & 0.217 \\
\hline \multicolumn{4}{|c|}{ 7-OH-CBD } \\
\hline $\mathrm{C}_{\max }(\mathrm{ng} / \mathrm{mL})$ & $159.93 \pm 101.75$ & $151.45 \pm 58.81$ & 0.727 \\
\hline $\mathrm{T}_{\max }($ hour$)$ & $1.0(0.5-2)$ & $1.5(1-2)$ & 0.194 \\
\hline $\mathrm{AUC}_{0-10 \mathrm{~h}}(\mathrm{ng} / \mathrm{mL} \cdot \mathrm{h})$ & $306.73 \pm 204.52$ & $280.00 \pm 128.62$ & 0.587 \\
\hline $\mathrm{AUC}_{0-24 \mathrm{~h}}(\mathrm{ng} / \mathrm{mL} \cdot \mathrm{h})$ & $327.54 \pm 217.36$ & $299.37 \pm 140.64$ & 0.586 \\
\hline $\mathrm{K}_{\mathrm{e}}\left(\mathrm{h}^{-1}\right)$ & $0.21 \pm 0.06$ & $0.26 \pm 0.09$ & 0.170 \\
\hline $\mathrm{t}_{1 / 2}(\mathrm{~h})$ & $3.51 \pm 0.94$ & $2.89 \pm 0.74$ & 0.118 \\
\hline \multicolumn{4}{|c|}{ 7-COOH-CBD ${ }^{\S}$} \\
\hline $\mathrm{C}_{\max }(\mathrm{ng} / \mathrm{mL})$ & $118.03 \pm 64.94$ & $74.73 \pm 31.84$ & 0.031 \\
\hline $\mathrm{T}_{\max }($ hour $)$ & $8.0(3-24)$ & $6.0(4-24)$ & 0.272 \\
\hline $\mathrm{AUC}_{0-10 \mathrm{~h}}(\mathrm{ng} / \mathrm{mL} \cdot \mathrm{h})$ & $885.94 \pm 531.41$ & $552.89 \pm 225.26$ & 0.036 \\
\hline $\mathrm{AUC}_{0-24 \mathrm{~h}}(\mathrm{ng} / \mathrm{mL} \cdot \mathrm{h})$ & $2122.73 \pm 1257.15$ & $1343.35 \pm 569.39$ & 0.034 \\
\hline
\end{tabular}

Conversely, 6- $\alpha-\mathrm{OH}$ CBD and 7-COOH CBD showed different serum pharmacokinetic profiles for the two investigated cannabis preparations (Table 1). Although $\mathrm{T}_{\max }$ was not statistically different after decoction and oil intake, significant differences were observed in mean peak concentrations $(6-\alpha-\mathrm{OH}$ $\mathrm{CBD} \mathrm{C}_{\max } 0.80 \pm 0.41 \mathrm{ng} / \mathrm{mL}$ for decoction and $0.42 \pm 0.18 \mathrm{ng} / \mathrm{mL}$ for oil and 7-COOH CBD $\mathrm{C}_{\max }$ $118.03 \pm 64.94$ for decoction and $74.73 \pm 31.84$ for oil) and in AUCs of both metabolites. Finally, it is worth noting that complete elimination of 7-COOH-CBD was not accomplished during the $24 \mathrm{~h}$ time of collection.

\subsection{Urinary Excretion of CBD Metabolites after Decoction and Oil Administration}

Following the administration of both herbal preparations, all the investigated metabolites were excreted in urine, although in significantly different total amounts (Figure 2).

At the first collection time interval ( $2 \mathrm{~h}$ after the start of experiment), all the investigated metabolites could be measured in urine samples. Apart from 6- $\alpha-\mathrm{OH}-\mathrm{CBD}$, which had significantly lower excretion after oil administration $(p<0.001)$, all the other metabolites were similarly eliminated in $24 \mathrm{~h}$ urine samples both after decoction and oil administration. 


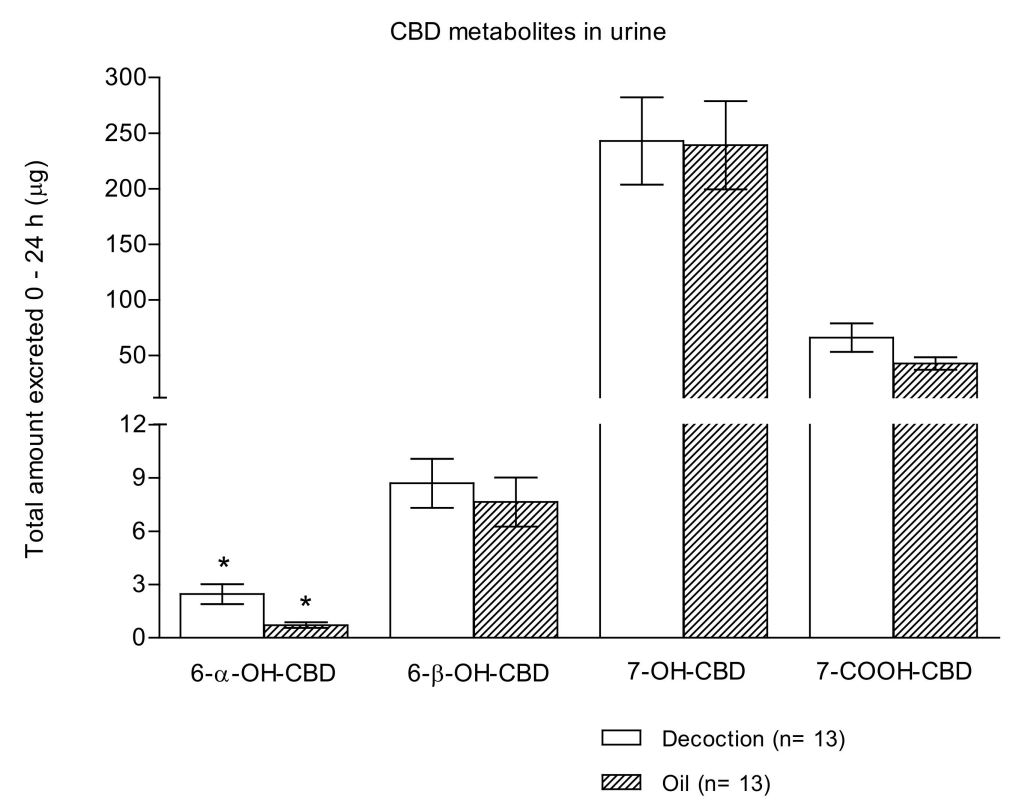

Figure 2. Total amount of 6- $\alpha-\mathrm{OH}-\mathrm{CBD}, 6-\beta-\mathrm{OH}-\mathrm{CBD}, 7-\mathrm{OH}-\mathrm{CBD}$, and 7-COOH-CBD excreted in $24 \mathrm{~h}$ urine samples following the administration of cannabis decoction and oil $(n=13$; mean \pm standard error). The symbol * shows significant differences between decoction and oil preparations values.

The most excreted CBD metabolite was 7-OH-CBD (mean \pm SD: $242.9 \pm 141.35 \mu \mathrm{g}$ in the case of decoction and $239.1 \pm 143.1 \mu \mathrm{g}$ in the case of oil). Furthermore, $7-\mathrm{COOH}-\mathrm{CBD}$ was the second most prevalent metabolite, about four time less concentrated than the previous one $(65.9 \pm 46.2 \mu \mathrm{g}$ and $42.7 \pm 20.3 \mu \mathrm{g}$ after decoction and oil consumption, respectively). Additionally, $6-\beta-\mathrm{OH}-\mathrm{CBD}$ was detected in urine samples at a concentration 30 times lower than that of the hydroxy metabolite $(8.7 \pm 4.9 \mu \mathrm{g}$ for decoction vs. $7.6 \pm 5.0 \mu \mathrm{g}$ for oil), and finally, $6-\alpha-\mathrm{OH}-\mathrm{CBD}$ was excreted in urine with concentrations two orders of magnitude less $(2.5 \pm 2.0 \mu \mathrm{g}$ after decoction intake and $0.7 \pm 0.6 \mu \mathrm{g}$ after oil intake).

\section{Discussion}

To the best of our knowledge, this is the first study on CBD metabolites' disposition in serum and urine samples of adult healthy volunteers after controlled administration of medical cannabis decoction and oil.

Analyzing the same samples, we recently reported the kinetics of THC, CBD, their acidic precursors, and THC metabolites. In that vein, we observed that even if cannabis oil contained a significantly higher amount of THC, its bioavailability and that of its metabolites was similar in both herbal preparations. Conversely, the THC acidic precursor was equivalently present in both preparations and correspondently absorbed in serum. Whereas oil contained a significantly higher amount of CBD and a lower amount of CBDA, absorption was significantly higher after decoction intake for both compounds. THC metabolites showed a later onset with respect to parent drug in the absorption phase and a slower decrease to baseline. Whereas 11-OH-THC presented similar disposition after administration of the two herbal preparations, THC-COOH and THC-COOH Gluc absorption was greater after decoction administration, in agreement with what was reported for the parent cannabinoid [12].

Considering previously observed different CBD $(1.2 \pm 0.6 \mathrm{ng} / \mathrm{mL}$ after decoction administration vs. $0.4 \pm 0.3 \mathrm{ng} / \mathrm{mL}$ after oil administration peaking at a mean of $2 \mathrm{~h}$ peak time $)$ and CBDA $(91.4 \pm 40.5 \mathrm{ng} / \mathrm{mL}$ after decoction administration vs. $28.3 \pm 17.4 \mathrm{ng} / \mathrm{mL}$ after oil administration at a mean of $1 \mathrm{~h}$ peak time) bioavailability between the two cannabis reparations at the same dose, CBD metabolites' disposition was expected to vary following decoction and oil administration. In reality, a different disposition was observed in serum only in the case of the least concentrated CBD metabolite, $6-\alpha-\mathrm{OH}-\mathrm{CBD}$, and the 
most concentrated one, 7-COOH-CBD, whose biosynthesis was significantly higher for decoction than for oil. In the case of 6- $\alpha-\mathrm{OH}-\mathrm{CBD}$, a higher formation following decoction intake was confirmed by a greater urinary excretion, which was not the case for 7-COOH-CBD, which showed a trend toward higher elimination after decoction consumption.

CBD metabolites' disposition following medical cannabis administration confirms that CBD is prevalently metabolized to the 7-carboxy metabolite, as already found by previous studies $[13,14]$. This metabolite was the most concentrated in serum but not the most excreted in urine, since at $24 \mathrm{~h}$ post experiment start, it was not yet totally eliminated from serum. Furthermore, taking into account the previously reported concentrations of CBD/CBDA [9], the current results demonstrate that concentrations of 7-COOH-CBD and 7-OH-CBD are more than 10 or 3 times higher than that of the parent substance, respectively.

There are few previous investigations on the pharmacokinetics of CBD metabolites in humans.

One early study established that after the administration of a of $20 \mathrm{mg}$ [3H]CBD dose to healthy volunteers by intravenous injection, 7-COOH-CBD was the most abundant metabolite in the plasma, while 7-OH-CBD was only a minor metabolite (the compounds were referred to as 11-carboxy-CBD and 11-hydroxy-CBD, respectively) [15].

More recently, the pharmacokinetic properties of CBD and some related metabolites were assessed after single- and multiple-dose administration of a highly purified pharmaceutical CBD preparation [14]. A single dose of 1500, 3000, 4500, or $6000 \mathrm{mg}$ was orally administered to four groups of healthy volunteers and $\mathrm{CBD}, 7-\mathrm{COOH}-\mathrm{CBD}$, and 6-OH-CBD were quantified in plasma samples. The reported metabolic profile was similar to the one here presented, with 7-COOH-CBD being the most prevalent serum metabolite, followed by 7-OH-CBD and 6-OH-CBD.

More than what they have shown, we measured 6-OH metabolite isomers, which displayed different concentration values both in serum and in urine. The $6-\beta-O H-C B D$ was the most prevalent in both investigated matrices after the administration of each different preparation.

The above-cited study indicated also that metabolites' $\mathrm{T}_{\max }$ in serum was around $4.6 \mathrm{~h}$, independent of the administered dose. Conversely, $\mathrm{T}_{\max }$ observed in our study was shorter $(1.5 \mathrm{~h})$ for all the metabolites apart from 7-COOH-CBD $(8 \mathrm{~h})$, without any difference between the two administered preparations. Although that and our investigation reached similar results concerning CBD metabolites disposition, the divergent $\mathrm{T}_{\max }$ values might be due to the presence of THC in the herbal decoction and the oil, while it was not present in the pharmaceutical CBD preparation [14]. Finally, in our study, a significant interindividual variability in CBD metabolites' concentration both in serum and urine was observed among the enrolled healthy subjects selected, in agreement of what previously reported in other studies on individuals treated with cannabis herbal preparations [9,11,12]. As a matter of fact, the most important limitations are indeed the inclusion of healthy volunteers, predominantly males, the very small number of tested individuals, and the low administered doses.

\section{Materials and Method}

\subsection{Subjects' Enrolment}

Twelve healthy males and two healthy females, were recruited for the two sessions of the study by "word of mouth" and the database of volunteers who had participated in previous studies at Clinical Pharmacology Unit, Hospital Universitari Germans Trias i Pujol, Badalona, Spain. Participants were informed of the characteristics of the study and signed an informed consent form. Eligibility criteria included social or recreational cannabis use in the last 12 months (from weekly to monthly to use, without criteria of cannabis use disorder) and oral cannabis use at least once in participants' life (e.g., cakes, cookies, oil, and/or infusion). Exclusion criteria were an history of any serious medical diseases or chronic medication use, any or psychopathological condition including substance use disorder (except for nicotine, according to the Diagnosis and Statistical Criteria for Mental Disorders, DSM-5), chronic medication use, and severe adverse reactions associated with cannabis use, pregnancy, and lactation. 
The participants underwent a general medical examination, including blood and urine analysis and a 12-lead electrocardiogram (ECG) within three weeks prior to inclusion and were given time to familiarize with study procedures and questionnaires. The study protocol was approved by the local Human Research Ethics Committee (CEIC-HGTiP, Badalona, Spain), and the study was conducted in accordance with the Declaration of Helsinki and Spanish laws concerning clinical research.

\subsection{Cannabis Decoction and Oil Preparation}

With the authorization of the Italian and Spanish Medicines Agencies, the Italian Military Pharmaceutical Chemical Factory in Florence provided us with a standardized medicinal cannabis flower cup extract called FM2 that contains 5.9\% THC and 8.4\% CBD. The preparation of the decoction and the oil was performed as previously reported $[9,13]$. A decoction volume of $1 \mathrm{~mL}$ and three drops of oil were saved for later analysis in order to verify the exact amount of THC and CBD administered.

\subsection{Study Design}

The study was open label, not randomized, and single blinded, since participants were aware of the cannabis preparation but not of the administered doses. The study included two separate experimental sessions, the first one for decoction administration and second one for oil administration, separated by at least 3 weeks. Further details were previously reported [9]. After an overnight fast, the subjects were admitted to the Clinical Research Unit (UPIC) at 07:45 a.m. Upon arrival of the subjects, they were asked about any substance/drug or event that could affect their participation in the study. The subjects had previously been instructed not to consume psychoactive drugs for at least the seven days prior to the study or products with caffeine and alcohol for the previous 24 and $48 \mathrm{~h}$, respectively.

Before starting each the of the two experimental sessions, a urine sample was collected for drug detection (Instant-View ${ }^{\circledR}$, Multipanel 10 Test Drug Screen, Alfa Scientific Designs Inc., Poway, CA, USA), and analysis of alcohol was performed in exhaled air (Dräger alcotest 5820, Dräger, Germany). If either of these two tests was positive, the subjects were excluded from the experimental session. The subjects remained sitting in bed in the Hospital's Clinical Research Unit (UPIC), throughout the session. The last cigarette allowed was $2 \mathrm{~h}$ before starting the session, smoking being prohibited during the course of the study. Subjects were placed on an intravenous catheter for the collection of blood samples, and sweat patches were applied on the back. The administration of decoction $(100 \mathrm{~mL})$ or oil ( 15 drops, $0.45 \mathrm{~mL}$ ) was carried out between $08: 15$ and 08:30 h. After the herbal preparations' administration, the mouth was washed twice with water to avoid oral contamination in oral fluid collection. Subjects received breakfast, lunch, and a snack two, five, and eight hours respectively, after administration. The duration of the experimental sessions was $10 \mathrm{~h}$, and subjects returned to the center at $24 \mathrm{~h}$ after administration. Other variables related to pharmacological effects were evaluated and reported in a previous publication [9].

\subsection{Biological Samples' Collection}

Serum $(2 \mathrm{~mL})$ from whole-blood centrifugation was collected from 15 min prior to administration (baseline, $0 \mathrm{~h}$ ) and $0.5,1,1.5,2,3,4,6,8,10$, and $24 \mathrm{~h}$ after decoction or oil administration. Urine was collected $15 \mathrm{~min}$ prior to administration (zero time) and then between $0-2,2-4,6-8,8-10$, and 10-24 $\mathrm{h}$ intervals after administration.

\subsection{Determination of Cannabinoids in Herbal Preparations and CDB Metabolites in Serum and Urine Samples}

THC, CBD, THCA-A, and CBDA were quantified in herbal preparations by ultra-high-performance liquid chromatography-tandem mass spectrometry (UHPLC-MS/MS) equipped with an electrospray ionization source operating in positive-ion mode as previously reported [15]. With respect to $6-\alpha-\mathrm{OH}-\mathrm{CBD}$, $6-\beta-\mathrm{OH}-\mathrm{CBD}, 7-\mathrm{OH}-\mathrm{CBD}$, and 7-COOH-CBD, 99\% pure standards were purchased from Dalton Research Molecules (Toronto, ON, Canada), and a specific UHPLC-MS-MS assay has been recently developed 
and validated with limits of quantification ranging from 0.05 to $0.1 \mathrm{ng} / \mathrm{mL}$. with average inter-/intra-day accuracy and precision $<15 \%$ [16].

\subsection{Statistical Analysis}

Maximum concentration $\left(\mathrm{C}_{\max }\right)$, time needed to reach maximum concentrations $\left(\mathrm{T}_{\max }\right)$, area under the concentration-time curve at 10 and $24 \mathrm{~h}\left(\mathrm{AUC}_{0-10 \mathrm{~h}}, \mathrm{AUC}_{0-24 \mathrm{~h}}\right)$, elimination half-life $\left(\mathrm{t}_{1 / 2}\right)$, and elimination constant ( $\mathrm{K}_{\mathrm{e}}$, calculated with at least three sample points) for CBD metabolites in serum were determined using Pharmacokinetic Functions for Microsoft Excel (Joel Usansky, Atul Desai, and Diane Tang-Liu, Department of Pharmacokinetics and Drug Metabolism, Allergan, Irvine, CA, USA at https://www.coursehero.com/file/30859156/pkfdoc/).

A Student's $t$-test for paired samples was performed to assess potential differences between pharmacokinetic parameters in the decoction and the oil, since a Kolmogorov-Smirnov test showed a normal parameter distribution. A nonparametric Wilcoxon signed-rank test for paired samples was instead applied for $\mathrm{T}_{\max }$, not a continuous variable and non-normally distributed.

The time course of analytes concentrations was analyzed employing a two-way, repeated-measures ANOVA with formulation and time $(0-10 \mathrm{~h})$ as factors. When treatment condition or the treatment condition $\times$ time interaction was statistically significant, a Student's $t$-test for paired samples was performed to compare analytes' concentrations in the decoction and oil. All statistical tests were performed at each time point using the PASW Statistics 18.0 (SPSS Inc., Chicago, IL, USA). A value of $p<0.05$ was considered statistically significant.

\section{Conclusions}

The present study aimed to characterize and compare the disposition of CBD metabolites in serum and urine after the controlled administration of medical cannabis decoction and oil. Notwithstanding the highlighted study limitations, the pharmacokinetic profile of the most representative CBD metabolites showed several differences between the decoction and the oil, in accordance with the different availability of CBD for the two formulations. Furthermore, the pharmacokinetic characterization of CBD metabolites is crucial for the clinical practice since cannabis herbal preparations are increasingly used for several pathological conditions.

Author Contributions: Conceptualization, R.P., M.F., S.P., and F.P.B.; methodology, A.P.P.-A., G.M., M.G., L.P., A.D.T., E.P., C.P.-M., and S.M.; resources, R.P., M.F., and F.P.B.; data curation, A.P.P.-A., G.M., M.G., L.P., E.P., C.P.-M., and S.M.; writing—original draft preparation, R.P., M.F., L.P., S.P., A.D.T., and F.P.B.; writing-review and editing, all authors. All authors have read and agreed to the published version of the manuscript.

Funding: The investigation was partially carried out with funding from the Presidency of the Ministers Council, Department of Antidrug Policy, and from the Department of Excellence SBSP, University "Politecnica delle Marche" of Ancona; and partially funded by grants from the Instituto de Salud Carlos III (ISCIII, Fondo Investigación sanitaria (FIS)-Fondo Europeo de Desarrollo Regional (FEDER), grant numbers: FIS PI14/00715 and PI20/00879, ISCIII-Red de Trastornos Adictivos RTA grant number: RD16/0017/0003, ISCIII-Plataforma de Unidades de Investigación y Ensayos Clínicos-SCREN, grant number: PT 17/0017/0016; AGAUR Gencat Suport Grups Recerca, grant number: 2017 SGR 316; Ministerio de Sanidad, Política Social e Igualdad (Plan Nacional Sobre Drogas-PNSD, grant number: 2015I054).

Acknowledgments: The authors thank Simonetta Di Carlo and Antonella Bacosi, Michele Sciotti, and Josuè Gottardi for technical assistance.

Conflicts of Interest: There are no potential conflicts of interest or any financial or personal relationships with other people or organizations that could inappropriately bias the conduct and findings of this study.

\section{References}

1. Pacifici, R.; Marchei, E.; Salvatore, F.; Guandalini, L.; Busardò, F.P.; Pichini, S. Evaluation of long-term stability of cannabinoids in standardized preparations of cannabis flowering tops and cannabis oil by ultra-high-performance liquid chromatography tandem mass spectrometry. Clin. Chem. Lab. Med. 2018, 56, 94-96. [CrossRef] [PubMed] 
2. Pacifici, R.; Marchei, E.; Salvatore, F.; Guandalini, L.; Busardò, F.P.; Pichini, S. Evaluation of cannabinoids concentration and stability in standardized preparations of cannabis tea and cannabis oil by ultra-high performance liquid chromatography tandem mass spectrometry. Clin. Chem. Lab. Med. 2017, 55, 1555-1563. [CrossRef] [PubMed]

3. Pacifici, R.; Pichini, S.; Pellegrini, M.; Rotolo, M.C.; Giorgetti, R.; Tagliabracci, A.; Busardò, F.P.; Huestis, M.A. THC and CBD concentrations in blood, oral fluid and urine following a single and repeated administration of "light cannabis". Clin. Chem. Lab. Med. 2019, 58, 682-689. [CrossRef] [PubMed]

4. Pacifici, R.; Marchei, E.; Salvatore, F.; Guandalini, L.; Busardò, F.P.; Pichini, S. Stability of cannabinoids in cannabis FM1 flowering tops and oil preparation evaluated by ultra-high performance liquid chromatography tandem mass spectrometry. Clin. Chem. Lab. Med. 2019, 57, e165-e168. [CrossRef] [PubMed]

5. Marchei, E.; Tittarelli, R.; Pellegrini, M.; Rotolo, M.C.; Pacifici, R.; Pichini, S. Is "light cannabis" really light? Determination of cannabinoids content in commercial products. Clin. Chem. Lab. Med. 2020, 58. [CrossRef] [PubMed]

6. Brunetti, P.; Pichini, S.; Pacifici, R.; Busardò, F.P.; Del Rio, A. Herbal preparations of medical cannabis: A vademecum for prescribing doctors. Medicina 2020, 56, 237. [CrossRef] [PubMed]

7. Huestis, M.A.; Solimini, R.; Pichini, S.; Pacifici, R.; Carlier, J.; Busardò, F.P. Cannabidiol adverse effects and toxicity. Curr. Neuropharmacol. 2019, 17. [CrossRef] [PubMed]

8. Brunetti, P.; Lo Faro, A.F.; Pirani, F.; Berretta, P.; Pacifici, R.; Pichini, S.; Busardò, F.P. Pharmacology and legal status of cannabidiol. Ann. Ist. Super. Sanita 2020, 56.

9. Pérez-Acevedo, A.P.; Pacifici, R.; Mannocchi, G.; Gottardi, M.; Poyatos, L.; Papaseit, E.; Pérez-Mañá, C.; Martin, S.; Busardò, F.P.; Pichini, S.; et al. Disposition of cannabinoids and their metabolites in serum, oral fluid, sweat patch and urine from healthy individuals treated with pharmaceutical preparations of medical cannabis. Phyther. Res. 2020. [CrossRef] [PubMed]

10. Barco, S.; Fucile, C.; Manfredini, L.; De Grandis, E.; Gherzi, M.; Martelli, A.; Tripodi, G.; Mattioli, F.; Cangemi, G. A UHPLC-MS/MS method for the quantification of $\Delta 9$-tetrahydrocannabinol and cannabidiol in decoctions and in plasma samples for therapeutic monitoring of medical cannabis. Bioanalysis 2018, 10, 2003-2014. [CrossRef] [PubMed]

11. Gherzi, M.; Milano, G.; Fucile, C.; Calevo, M.G.; Mancardi, M.M.; Nobili, L.; Astuni, P.; Marini, V.; Barco, S.; Cangemi, G.; et al. Safety and pharmacokinetics of medical cannabis preparation in a monocentric series of young patients with drug resistant epilepsy. Complement. Ther. Med. 2020, 51. [CrossRef] [PubMed]

12. Pellesi, L.; Licata, M.; Verri, P.; Vandelli, D.; Palazzoli, F.; Marchesi, F.; Cainazzo, M.M.; Pini, L.A.; Guerzoni, S. Pharmacokinetics and tolerability of oral cannabis preparations in patients with medication overuse headache (MOH)—A pilot study. Eur. J. Clin. Pharmacol. 2018, 74, 1427-1436. [CrossRef] [PubMed]

13. Ujváry, I.; Hanuš, L. Human metabolites of cannabidiol: A review on their formation, biological activity, and relevance in therapy. Cannabis Cannabinoid Res. 2016, 1, 90-101. [CrossRef] [PubMed]

14. Taylor, L.; Gidal, B.; Blakey, G.; Tayo, B.; Morrison, G. A phase I, randomized, double-blind, placebo-controlled, single ascending dose, multiple dose, and food effect trial of the safety, tolerability and pharmacokinetics of highly purified cannabidiol in healthy subjects. CNS Drugs 2018, 32, 1053-1067. [CrossRef] [PubMed]

15. Wall, M.; Brine, D.; Perez-Reyes, M. Metabolism of cannabinoids in man. In Tea Pharmacology of Marijuana; Braude, M.C., Szara, S., Eds.; Raven Press: New York, NY, USA, 1976; pp. 93-113.

16. Pichini, S.; Malaca, S.; Gottardi, M.; Pérez-Acevedo, A.P.; Papaseit, E.; Perez-Maña, C.; Farré, M.; Pacifici, R.; Tagliabracci, A.; Mannocchi, G.; et al. UHPLC-MS/MS analysis of cannabidiol metabolites in serum and urine samples. Application to an individual treated with medical cannabis. Talanta 2021, 223, 121772. [CrossRef] [PubMed]

Publisher's Note: MDPI stays neutral with regard to jurisdictional claims in published maps and institutional affiliations.

(C) 2020 by the authors. Licensee MDPI, Basel, Switzerland. This article is an open access article distributed under the terms and conditions of the Creative Commons Attribution (CC BY) license (http://creativecommons.org/licenses/by/4.0/). 